\title{
Assessment of dental caries risk in children based on color Doppler US and the changes in blood perfusion in the salivary glands during salivary stimulation
}

\author{
Ahmet Tuncay Turgut, Melek Dilek Turgut, Meryem Tekçiçek, Pınar Koşar, Pınar Özdemir, Uğur Koşar, \\ Vikram Dogra
}

\begin{abstract}
PURPOSE
To investigate in otherwise healthy children the association between the caries index, the stimulated salivary flow rate (SFR), and the spectral Doppler findings of the changes in blood perfusion in the salivary glands during the secretion of saliva.

MATERIALS AND METHODS

The study group consisted of 38 children with a mean age of $9.47 \pm 1.89$ years. The caries index was calculated by determining the number of decayed, missing, and filled teeth. Groups A, B, and C represented subjects with low, moderate, and normal SFRs, respectively, calculated by obtaining chewing-stimulated whole saliva. All subjects were examined by color Doppler ultrasonography (CDUS) before and during secretory stimulation with lemon, by which maximum systolic velocity (MSV), pulsatility index $(\mathrm{PI})$, resistive index (RI), and flow volume (FV) were calculated at the external carotid and facial arteries.

RESULTS

The differences for spectral indices obtained before and after stimulation were significantly different among Groups $A, B$, and $C$ at the external carotid artery $(P=0.006$ for delta MSV, $P=0.014$ for delta $\mathrm{PI}, P<0.001$ for delta $\mathrm{RI}$, and $P=0.022$ for delta FV) and at the facial artery $(P=0.001$ for delta MSV, $P$ $=0.004$ for delta $\mathrm{PI}, P<0.001$ for delta $\mathrm{RI}$, and $P<0.001$ for delta FV). In addition, significant correlations were calculated between the SFR and the aforementioned delta values.

CONCLUSION

CDUS enabled the evaluation of changes in blood perfusion in the salivary glands during salivary stimulation and may be a promising tool for the assessment of caries risk in children.

Key words: $\bullet$ Doppler ultrasonography $\bullet$ saliva $\bullet$ dental caries - risk
\end{abstract}

D ental caries is not only the most common infectious disease of the oral cavity, but it is also the single most common chronic childhood disease $(1,2)$. The onset and progression of caries are closely associated with internal defense factors, such as saliva, tooth surface morphology, and general health as well as a number of external factors, such as diet, oral hygiene, and fluoride availability (3). Although this disease is very common, the underlying mechanisms of the variable susceptibility of children to caries have been the topic of interest in the field of dental research for decades.

Among various bacteriologic and nonbacteriologic criteria for identifying individuals at high risk for dental caries, salivary parameters are of significant relevance for assessing caries activity. It is generally accepted that whole saliva, secreted by the major and minor salivary glands, and its components are critical for the maintenance of the integrity of the teeth and oral soft tissues (4). The main salivary parameters associated with an increased risk for caries are buffer capacity, cariogenic bacteria counts, $\mathrm{pH}$, flow rate, and total protein content $(5,6)$. In this regard, the knowledge of normal salivary composition, flow, and functional properties may permit a better assessment of dental caries susceptibility. Some studies have seemed to support the validity of a combined evaluation of the decayed, missing, and filled teeth (DMFT) index, pH, salivary flow, and buffer power of saliva in the prediction of caries $(4,7,8)$. However, salivary flow rate (SFR) is undoubtedly the most important single parameter because the cariostatic activity or efficacy of practically all other salivary parameters depends on the flow rate (9).

Importantly, an adequate supply of saliva is critical to the preservation and maintenance of oral tissue. In this regard, decreased salivary secretion, which usually manifests with clinical findings such as xerostomia, has been noted to be closely associated with increased dental caries (10, 11). Nevertheless, an overwhelming majority of children suffering from caries do not have clinical presentations involving the apparently subjective feeling of xerostomia. Although the measurement of stimulated salivary flow has been reported to be important only when it is greatly reduced, as occurs, for example, in xerostomy, the finding of an almost normal flow has not yet been found to be sufficient on its own for the diagnosis of carioreceptivity (8). Conversely, normal values exhibit large biological variations and are difficult to define for all age groups and both genders $(12,13)$. In this regard, little attention has been paid to the variability of normal salivary flow and function and its impact on dental health, although much has been written on the relationship between salivary hypofunction and caries (14).

Color Doppler ultrasonography (CDUS) is a well-established technique for analyzing the vascularity of the salivary glands and for characterizing some pathologic conditions (15). In animal models, a 
relationship between changes in salivary gland arterial inflow and salivary secretion has been shown $(16,17)$. Based on these studies, it is known that blood flow and cholinergic tone can influence salivary gland secretion. Moreover, it has been noted in physiological studies that intense hyperemia accompanies the secretion of saliva, with blood perfusion of the salivary glands increasing more than fivefold over the basal value of 0.1-0.6 $\mathrm{mL} / \mathrm{min}$ (18). In the current study, we aimed to define, for the first time in the pediatric population, the role of CDUS in the assessment of physiological changes in blood perfusion occurring in the salivary glands during the secretion of saliva. More importantly, we aimed to investigate the association in otherwise healthy children between the aforementioned sonographic changes and stimulated SFR and the DMFT index, which would be helpful in establishing a basis on which to develop a system for caries risk assessment. To the best of our knowledge, this is the first study that investigates the utility of a radiological method for the relevant assessment.

\section{Materials and methods}

Thirty-eight children ( 20 boys and 18 girls) with a mean age of $9.47 \pm 1.89$ years (age range, 6-14 years) were recruited randomly from the patients referred for routine dental examinations. The exclusion criteria were oral mucosal alterations, upper respiratory tract infections, endocrine, pulmonary, renal, hepatic or autoimmune diseases, a history of vascular disease, and a history of treatment with any drug that could affect the vascular and/or salivary flow, such as diuretics or anti-histamines. The subjects and their parents were questioned regarding complaints associated with xerostomia, and none had such complaints. Informed consent was obtained from all parents to allow a dental examination, saliva collection and CDUS examination, after explaining the study protocol to the parent and child in detail. The study protocol was approved by the local institutional ethics committee.

Every child underwent an oral examination performed by a skilled pediatric dentist (M.T.) using a noninvasive technique (mouth mirror, dental probe, and cotton roll). The type of dentition was either mixed (concomitant presence of primary and permanent teeth) or permanent. Caries was determined visually with optimal illumination of the oral cavity, in which the detection of an apparent carious cavity on visual inspection was defined as a caries lesion. The prevalence of caries was calculated by determining the number of decayed (d, D), missing ( $m, M)$, and filled ( $\mathrm{f}, \mathrm{F})$ teeth $(t, T)$. Notably, teeth that were lost because of trauma or exfoliation were excluded from the relevant calculation. Accordingly, the frequency of caries was assessed based on the DMFT score for permanent teeth and the dmft score for primary teeth, which were used to assess caries experience. For analysis, the subjects were divided into low and high caries risk groups (Group 1 and Group 2, respectively), based on the sum of the respective DMFT and dmft scores for the permanent and primary teeth. Group 1 consisted of 22 children with $<5$ for the combined DMFT and dmft scores. Group 2 consisted of 16 children with $\geq 5$ for the combined DMFT and dmft scores. The reason for choosing a DMFT/dmft scores of 5 as the cut-off between the high and low groups was that the mean of the combined DMFT and dmft scores for the study group was $4.89 \pm 3.51$.

The SFR was calculated by a skilled pediatric dentist (M.D.T.) by obtaining chewing-stimulated whole saliva, which was collected between 2 and 5 p.m. to minimize the effects of circadian variances (19). The subjects were asked to refrain from eating, drinking, and performing oral hygiene procedures for two hours before the test. Seated in the dental chair, the subjects were allowed to relax for a few minutes. The subjects were then asked to chew on $1 \mathrm{~g}$ of unflavored paraffin wax for $6 \mathrm{~min}$. After swallowing the saliva in the first minute, the subjects were instructed to spit the accumulated saliva periodically into a discard cup for the next five minutes. The SFR was then calculated from the collected saliva volume within a discarded syringe. The SFR results were expressed as $\mathrm{mL} / \mathrm{min}$ ). For analysis, SFRs were scored from 0 to 2:0 represented less than $0.7 \mathrm{~mL} / \mathrm{min}$; 1 represented from 0.7 to $0.9 \mathrm{~mL} / \mathrm{min}$; and 2 represented greater than $0.9 \mathrm{~mL} / \mathrm{min}$. Accordingly, Groups A $(n=17), B \quad(n=10)$, and C $(n=11)$ were defined as subjects with
SFR scores of 0,1 , and 2 representing low, moderate, and normal SFRs, respectively.

CDUS examinations were performed between 2 and 5 p.m. by an experienced radiologist (A.T.T.) who was blinded to the DMFT/dmft scores and SFRs of the subjects. For each examination, a CDUS scanner (Xario, Toshiba, Tokyo, Japan) equipped with a wide-band 5-12 $\mathrm{MHz}$ linear array transducer was used. All subjects were examined by CDUS before and during secretory stimulation with lemon, by which spectral waveform analysis was performed on the external carotid and facial arteries to assess the physiologic changes in blood inflow to the parotid and submandibular glands, respectively. Based on the fact that sonographic changes during salivary stimulation occur with similar features in all salivary glands (15) and that in a previous study no significant differences were detected between the right and left submandibular glands for the differences between prestimulation and poststimulation spectral Doppler indices (18), only the arteries on the left side were examined. Each subject was examined in the supine position, with the neck hyperextended and the head turned slightly to the opposite side. The CDUS settings were adjusted dynamically to optimize visualization of the aforementioned arteries while avoiding artificial noise. For spectral waveform measurements, the Doppler angle was maintained at $60 \%$ or less. Salivary stimulation was performed by having the subjects suck on a slice of lemon and swallow the lemon juice after retaining it in the mouth for approximately $15 \mathrm{~s}$. During the act of sucking, care was taken to limit as much as possible movements of the mouth and face that could disturb the examination. The transducer was held in the same position during the procedure. For each patient, two spectral traces, one before and one during lemon juice administration were recorded from the aforementioned arteries. In each subject, maximum systolic velocity (MSV), pulsatility index (PI), resistivity index (RI) and flow volume (FV) were automatically calculated by the ultrasonography machine by analysis of the velocity waveforms. Delta values for each of the above spectral parameters were defined as the difference between the 

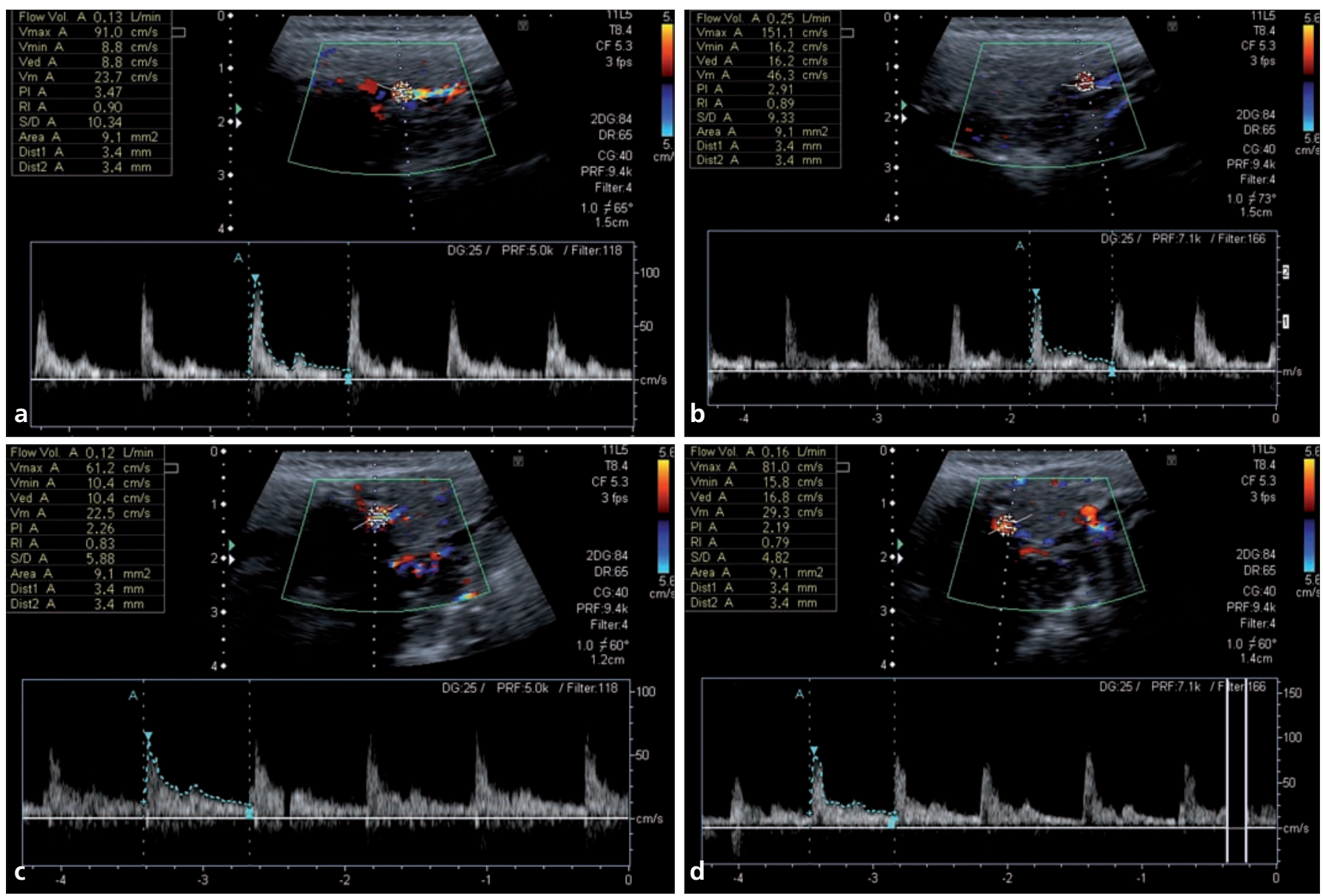

Figure 1. a-d. A 12-year-old girl with a combined dmft+DMFT score of 8 and a stimulated salivary flow rate of $0.4 \mathrm{~mL} / \mathrm{min}$. The analysis of the spectral waveforms obtained by CDUS of the external carotid $(\mathbf{a}, \mathbf{b})$ and facial $(\mathbf{c}, \mathbf{d})$ arteries before and during lemon stimulation revealed a slight increase of MSV and FV with an accompanying decrease of PI and RI.

values calculated before and after the lemon stimulation.

Statistical analysis was performed with a commercially available software (Statistical Package for Social Sciences, version 15, SPSS Inc., Chicago, Illinois, USA). For descriptive statistics, median (inter quartile range [IQR]) values were calculated for skewed continuous data, and means \pm standard deviations were determined for normally distributed variables, whereas percentage values were used for categorical data. For statistical analysis, the Mann-Whitney U test and Kruskal-Wallis test were used for determining the differences among the groups for skewed continuous data, and an independent samples ttest was used for normally-distributed variables, while a chi-square test was used for categorical data. Correlations between the values for the study variables were investigated by Pearson's or Spearman's correlation coefficient. Wilcoxon's signed ranks test or a paired t-test was used to test the significance of any change. A value of $P<0.05$ was considered statistically significant.

\section{Results}

In Group 1, the numbers of subjects with SFR scores of 0,1 , and 2 were 11,4 , and 7 , respectively. In Group 2 , the numbers of subjects with the aforementioned scores were 6,6 , and 4 , respectively. No significant differences were detected between Groups 1 and 2 for the number of subjects with respective SFR scores $(P>0.05)$. The mean SFR scores for the subjects in Group 1 and Group 2 were $0.81 \pm 0.42$ and $0.69 \pm 0.30$, respectively. No significant differences were detected between Group 1 and Group 2 for the mean SFR scores $(P>0.05)$. The numbers of subjects with low caries risk in Groups $\mathrm{A}$, $B$, and $C$ were 11,4 , and 7 , respectively, whereas the numbers of the subjects with high caries in these groups were 6,6 , and 4 , respectively. No significant differences were detected among
Groups $\mathrm{A}, \mathrm{B}$, and $\mathrm{C}$ for the numbers of subjects with low and high caries risks $(P>0.05)$. Conversely, the mean combined dmft+DMFT scores for Groups $\mathrm{A}, \mathrm{B}$, and $\mathrm{C}$ were $4.18 \pm 2.24,6.30 \pm 4.47$, and $4.73 \pm 4.08(P>0.05)$, respectively. No significant differences were detected among Groups $\mathrm{A}, \mathrm{B}$, and $\mathrm{C}$ for the mean combined $\mathrm{dmft}+\mathrm{DMFT}$ scores $(P$ $>0.05$ ). When Groups B and C were combined to represent subjects without low SFRs, no significant differences were detected between Group A and the combined Group $\mathrm{B}+\mathrm{C}$ for the mean combined $\mathrm{dmft}+\mathrm{DMFT}$ scores, which were $4.18 \pm 2.24$ and $5.48 \pm 2.24$, respectively $(P>0.05)$.

After the stimulation of salivary secretion, increases in MSV and FV, as well as decreases in PI and RI for the external carotid and facial arteries, which are waveform changes that are indicative of increased blood flow to the parotid and submandibular glands, respectively, were detected (Fig. 1 and 2). Changes in the above parameters 

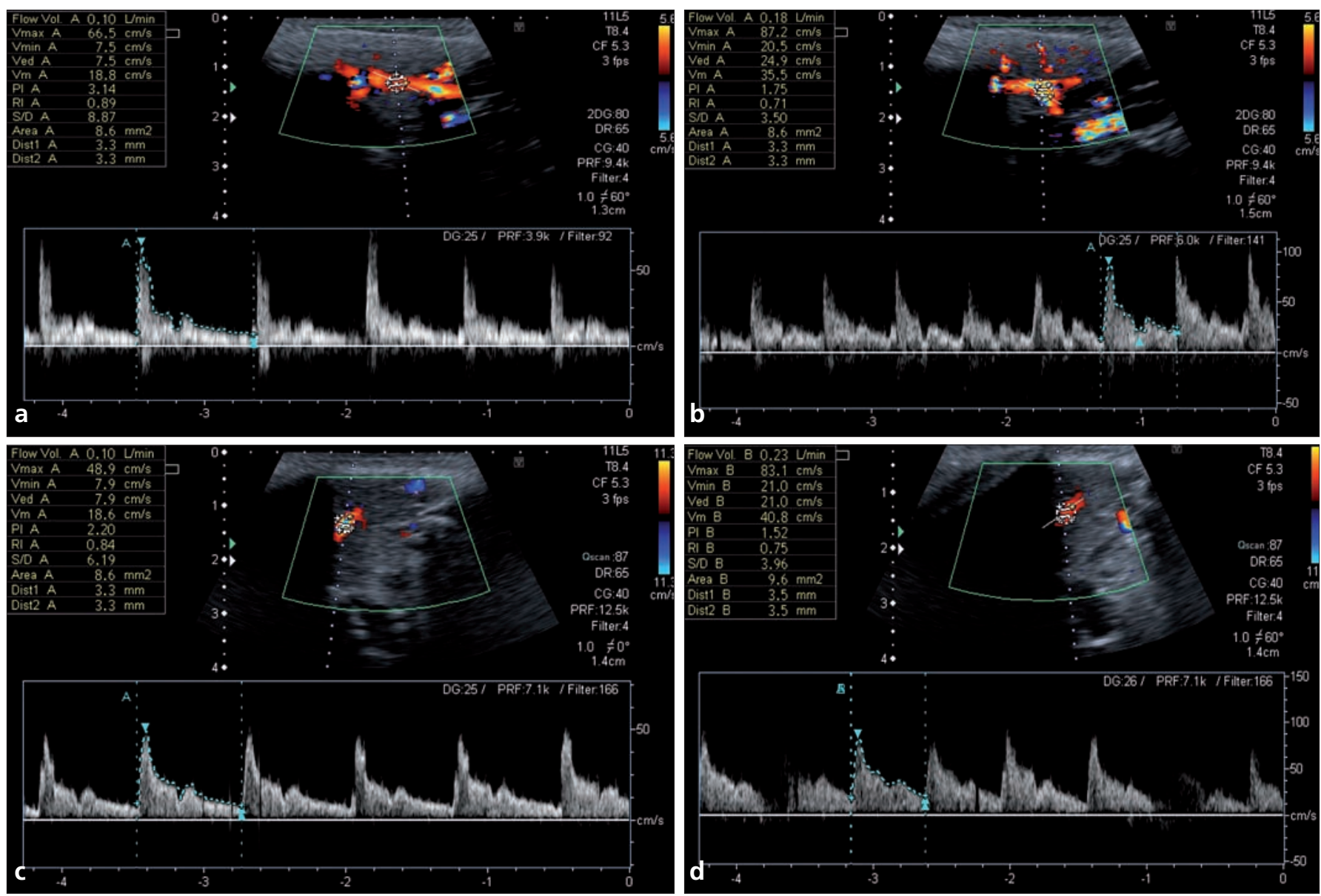

Figure 2. a-d. A 13-year-old boy with a combined dmft+DMFT score of 1 and a stimulated salivary flow rate of $1.2 \mathrm{~mL} / \mathrm{min}$. The analysis of the spectral waveforms obtained by CDUS of the external carotid $(\mathbf{a}, \mathbf{b})$ and facial $(\mathbf{c}, \mathbf{d})$ arteries before and during lemon stimulation revealed a marked increase of MSV and FV with an accompanying decrease of PI and RI.

were transient and tended to normalize within 15-20 s after the lemon juice was swallowed. The mean values for MSV, PI, RI, and FV, derived from the analysis of the waveforms obtained at the external carotid and facial arteries before and during the lemon stimulation, and the mean delta values for the aforementioned spectral parameters are given in Table 1. Accordingly, the paired differences for the means of MSV, PI, RI, and FV at the external carotid and facial arteries showed statistically significant differences $(P \leq 0.001$ for each). The median values in Groups 1 and 2 for the delta values of MSV, $\mathrm{PI}, \mathrm{RI}$, and FV at the external carotid and facial arteries are given in Table 2. Accordingly, a statistically significant difference was detected between Groups 1 and 2 for the means of the delta PI values obtained at the external carotid artery $(P=0.012)$. Furthermore, the difference between Groups 1 and 2 for delta MSV at the external carotid artery was calculated to be marginally statistically significant $(P=0.051)$. The median values in Groups A, B, and C for the delta values of MSV, PI, RI, and FV at the external carotid and facial arteries are given in Table 3, and Fig. 3 and 4. Accordingly, the aforementioned delta values obtained at the external carotid artery were significantly different among Groups $\mathrm{A}, \mathrm{B}$, and $\mathrm{C}$ $(P=0.006$ for delta MSV, $P=0.014$ for delta PI, $P<0.001$ for delta RI, and $P=$ 0.022 for delta FV). Likewise, the aforementioned delta values obtained at the facial artery were significantly different among Groups $\mathrm{A}, \mathrm{B}$, and $\mathrm{C}(P=0.001$ for delta MSV, $P=0.004$ for delta PI, $P$ $<0.001$ for delta RI, and $P<0.001$ for delta FV).

Statistically significant correlations were calculated between the combined dmft and DMFT scores and delta values for MSV and PI, obtained at the external carotid artery $(\mathrm{r}=0.40, P$ $=0.014$ and $\mathrm{r}=0.34, P=0.04$, respectively) (Figs. 5 and 6). Statistically significant correlations were calculated between the SFR values and delta values for MSV, PI, RI, and FV obtained at the external carotid artery $(\mathrm{r}=0.51, P=$ 0.001 for delta MSV, $\mathrm{r}=0.38, P=0.018$ for delta $\mathrm{PI}, \mathrm{r}=0.76, P<0.001$ for delta RI and $\mathrm{r}=0.36, P=0.026$ for delta FV). Furthermore, statistically significant correlations were calculated between the SFR and delta values for MSV, PI, RI and FV obtained at the facial artery $(\mathrm{r}=0.65, P<0.001$ for delta MSV, $\mathrm{r}=0.59, P<0.001$ for delta PI, $\mathrm{r}=0.74$, $P<0.001$ for delta RI and $\mathrm{r}=0.70, P<$ 0001 for delta FV). Between the external carotid and facial arteries, the correlations for the delta values for MSV, $\mathrm{PI}$, and RI values were also statistically significant $(\mathrm{r}=0.52, P=0.001$ for delta MSV, $\mathrm{r}=0.45, P=0.005$ for delta PI and $\mathrm{r}=0.76, P<0.001$ for delta RI).

\section{Discussion}

Dental caries is obviously still a major problem in children, and an improved approach to prevention and therapy is needed. In Turkey, the prevalence of 
Table 1. Comparison of the mean values ( \pm standard deviation) for spectral parameters calculated at the external carotid and facial arteries before and after stimulation and the mean delta values for the paired differences

\begin{tabular}{lcccc}
\hline Spectral parameter & Before stimulation & During stimulation & Delta value & $P$ \\
\hline MSVP & $82.56 \pm 19.54$ & $111.87 \pm 31.31$ & $29.31 \pm 22.04$ & $\leq 0.001$ \\
PIP & $2.81 \pm 0.46$ & $1.97 \pm 0.37$ & $0.84 \pm 0.34$ & $\leq 0.001$ \\
RIP & $0.89 \pm 0.03$ & $0.81 \pm 0.05$ & $0.07 \pm 0.04$ & $\leq 0.001$ \\
FVP & $103.65 \pm 31.26$ & $163.73 \pm 37.38$ & $60.08 \pm 33.71$ & $\leq 0.001$ \\
MSVS & $57.28 \pm 14.78$ & $79.00 \pm 23.55$ & $21.72 \pm 15.60$ & $\leq 0.001$ \\
PIS & $2.27 \pm 0.30$ & $1.57 \pm 0.31$ & $0.70 \pm 0.32$ & $\leq 0.001$ \\
RIS & $0.82 \pm 0.04$ & $0.74 \pm 0.06$ & $0.09 \pm 0.05$ & $\leq 0.001$ \\
FVS & $51.96 \pm 21.20$ & $88.01 \pm 41.26$ & $36.05 \pm 28.03$ & $\leq 0.001$ \\
\hline
\end{tabular}

MSVP, maximum systolic velocity at external carotid artery; PIP, pulsatility index at external carotid artery; RIP, resistive index at external carotid artery; FVP, flow volume at external carotid artery; MSVS, maximum systolic velocity at facial artery; PIS, pulsatility index at facial artery; RIS, resistive index at facial artery; FVS, flow volume at facial artery.

Table 2. Comparison of Groups 1 and 2 for the median (IQR) differences between the spectral parameters calculated at the external carotid and facial arteries before and during stimulation

\begin{tabular}{lccc}
\hline Spectral parameter & Group 1 & Group 2 & $P$ \\
\hline Delta MSVP & $33.73(23.05)$ & $23.24(19.65)$ & 0.051 \\
Delta PIP & $0.99(0.28)$ & $0.73(0.33)$ & 0.012 \\
Delta RIP & $0.07(0.05)$ & $0.07(0.03)$ & $>0.05$ \\
Delta FVP & $61.39(29.68)$ & $59.13(37.03)$ & $>0.05$ \\
Delta MSVS & $23.65(18.98)$ & $19.07(9.10)$ & $>0.05$ \\
Delta PIS & $0.74(0.35)$ & $0.64(0.29)$ & $>0.05$ \\
Delta RIS & $0.09(0.06)$ & $0.08(0.04)$ & $>0.05$ \\
Delta FVS & $36.19(29.95)$ & $35.85(26.11)$ & $>0.05$ \\
\hline
\end{tabular}

IQR, interquartile range; delta MSVP, difference for maximum systolic velocity at external carotid artery; delta PIP, difference for pulsatility index at external carotid artery; delta RIP, difference for resistive index at external carotid artery; delta FVP, difference for flow volume at external carotid artery; delta MSVS, difference for maximum systolic velocity at facial artery; delta PIS, difference for pulsatility index at facial artery; delta RIS, difference for resistive index at facial artery; delta FVS, difference flow volume at facial artery.

Table 3. Comparison of Groups A, B, and C for the median (IQR) differences between the spectral parameters calculated at the external carotid and facial arteries before and during stimulation

\begin{tabular}{lcccc}
\hline Spectral parameter & Group A & Group B & Group C & $P$ \\
\hline Delta MSVP & $19.53(13.36)$ & $27.10(20.62)$ & $46.45(25.25)$ & 0.006 \\
Delta PIP & $0.65(0.34)$ & $1.02(0.27)$ & $0.97(0.23)$ & 0.014 \\
Delta RIP & $0.04(0.02)$ & $0.07(0.02)$ & $0.11(0.04)$ & $<0.001$ \\
Delta FVP & $50.55(45.09)$ & $59.64(15.75)$ & $75.20(17.97)$ & 0.022 \\
Delta MSVS & $13.74(8.45)$ & $28.41(22.89)$ & $27.98(10.85)$ & 0.001 \\
Delta PIS & $0.54(0.36)$ & $0.74(0.08)$ & $0.92(0.30)$ & 0.004 \\
Delta RIS & $0.05(0.02)$ & $0.09(0.03)$ & $0.14(0.05)$ & $<0.001$ \\
Delta FVS & $18.81(17.96)$ & $51.35(10.14)$ & $48.76(23.40)$ & $<0.001$ \\
\hline
\end{tabular}

IQR, interquartile range; delta MSVP, difference for maximum systolic velocity at external carotid artery; delta PIP, difference for pulsatility index at external carotid artery; delta RIP, difference for resistive index at external carotid artery; delta FVP, difference for flow volume at external carotid artery; delta MSVS, difference for maximum systolic velocity at facial artery; delta PIS, difference for pulsatility index at facial artery; delta RIS, difference for resistive index at facial artery; delta FVS, difference flow volume at facial artery. dental caries in 5-, 12-, and 15-year-old children has been reported to be $70 \%$, $66 \%$, and $70 \%$, respectively, implying that large groups of children still suffer from extensive, untreated dental damage (20). Caries management by risk assessment is now receiving considerable attention. In this regard, several studies have attempted to determine risk factors that can be reliably used to assess the level of risk of caries progression in individual patients. However, there is no definitive formula currently available for this risk assessment, although studies are still under way.

DMFT on its own does not determine with any degree of certainty the future caries index; it can label an individual as carioactive or evaluate his or her past "experience of caries". Conversely, increased caries experience may be associated with a reduced ability to produce saliva (13). In the current study, the subjects with low DMFT scores had higher SFRs compared to those patients with high DMFT scores, although the difference was not statistically significant. One explanation for this finding may be that the aforementioned risk factors, other than SFR, might also have influenced the calculated caries risk. In addition, stimulated SFR, which contributes as much as $80 \%$ to $90 \%$ of the average daily salivary production (14), is often selected as a parameter for testing salivary gland function due to its good reproducibility $(21,22)$. Many years ago, Younger et al. (23) demonstrated that patients with caries had lower rates of stimulated saliva secretion, compared to healthy controls. In another study by Bardow et al. (24), subjects with low unstimulated SFRs and high rates of tooth demineralization were shown to have low stimulated SFRs, with a significant correlation between the two rates. Taking into account these data, stimulated SFR was used to investigate the caries risk for the subjects included in the current study.

The determination of a normal range of SFRs may enable the identification and proper management of individuals with values outside this range. Specifically, the clinical hallmark of hyposalivation for stimulated saliva has been described as an SFR less than $0.70 \mathrm{~mL} / \mathrm{min}(9,25,26)$. However, salivary hypofunction is difficult to assess, and the establishment of standard values has been impaired, mainly because 


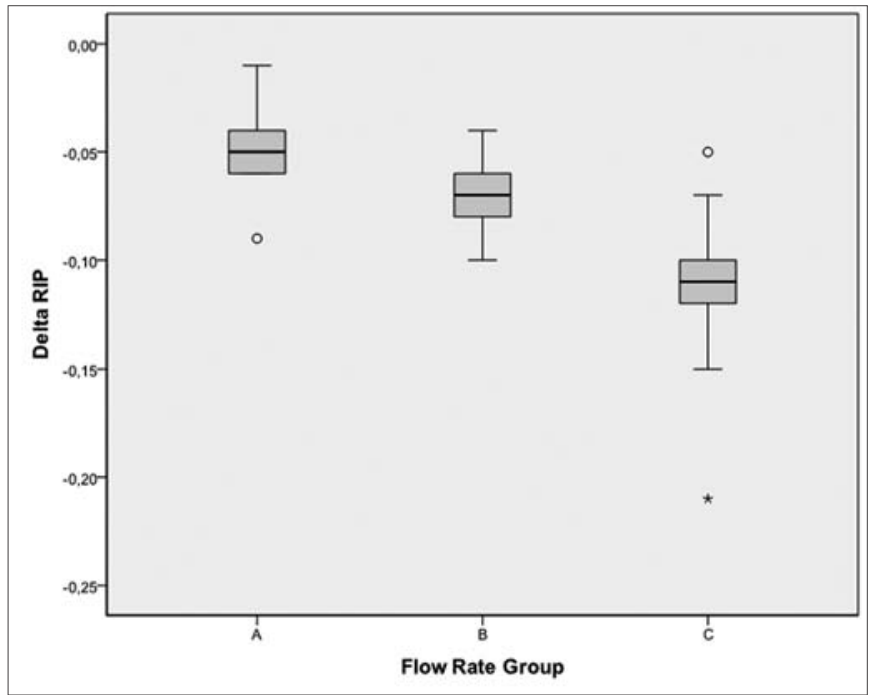

Figure 3. Box plot demonstrating the distribution of delta PI values obtained from the external carotid artery in Groups A, B, and C, defined according to the degree of salivary flow rate. A statistically significant difference was detected between the groups for the relevant variable $(P=0.014)$.

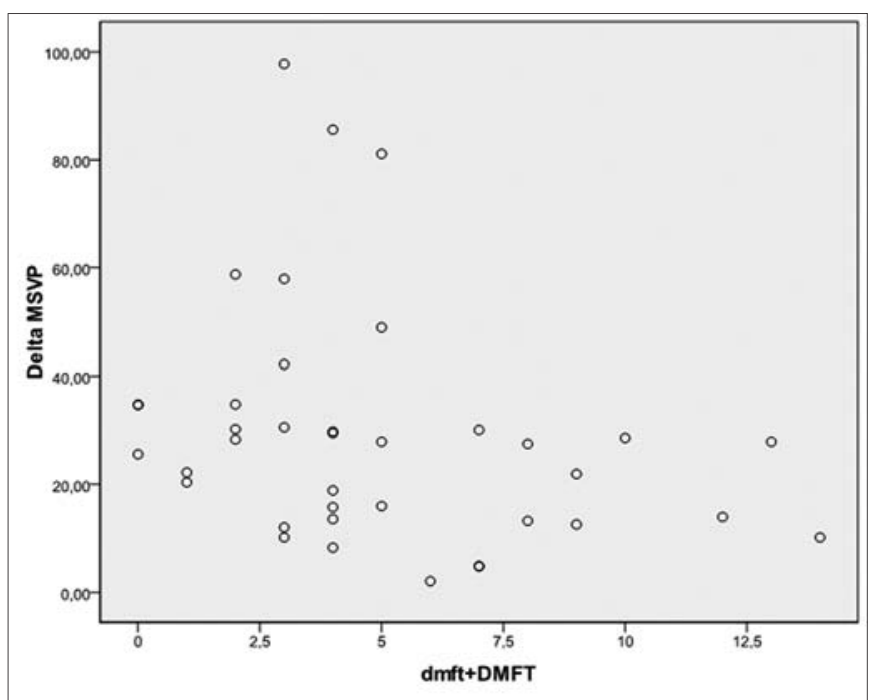

Figure 5. Scatter plot demonstrating the association between the delta MSV values obtained by CDUS examination of the external carotid artery and the combined dmft+DMFT scores of the subjects. The association between the two variables was statistically significant $(r=0.40, P=0.014)$.

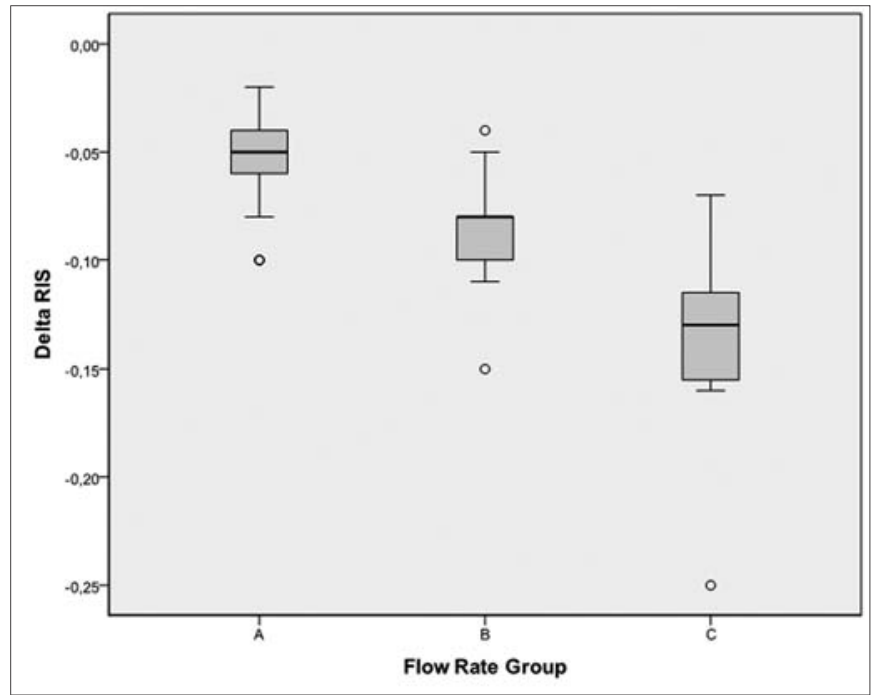

Figure 4. Box plot demonstrating the distribution of delta RI values obtained from the facial artery in Groups A, B, and C, defined according to the degree of salivary flow rate. A statistically significant difference was detected between the groups for the relevant variable $(P<0.001)$.

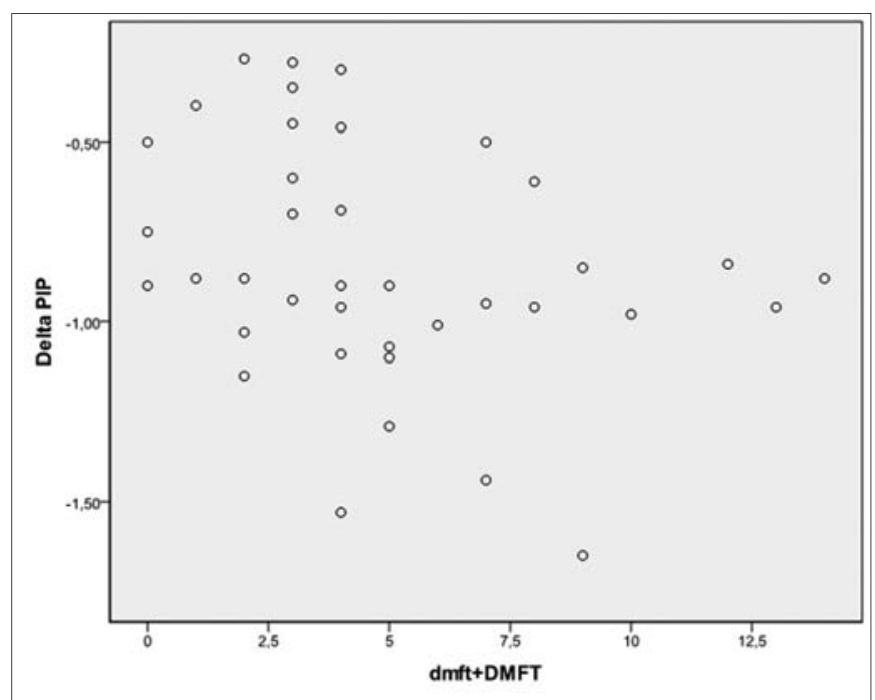

Figure 6. Scatter plot demonstrating the association between the delta PI values obtained by CDUS examination of the external carotid artery and the combined dmft+DMFT scores of the subjects. The association between the two variables was statistically significant $(\mathrm{r}=0.34, P=0.04)$ of the large variability in SFRs within and among individuals (14). Recently, a variation of $27 \%-44 \%$ was reported to be present for SFRs over time (27). Accordingly, a $45 \%$ range in stimulated parotid and submandibular SFRs was noted to be a normal variation, and values less than $45 \%$ were suggested to define salivary hypofunction (27). Therefore, the diagnosis and assessment of salivary hypofunction are relatively subjective, unless an individual base record of salivary flow has been established (14). It is evident that the aforementioned literature's data limit the utility of SFR as a screening tool for the prediction of caries risk.

In another study by Bardow et al. (24), subjects with low unstimulated SFRs and high rates of tooth demineralization were shown to have low stimulated SFRs, with a significant correlation between the two rates. Nevertheless, the authors also noted that the patients with normal SFRs, in addition to those with hyposalivation, had tooth demineralization rates greater than the mean mineral loss level calculated for all subjects included in the study, implying that hyposalivation is not a requirement for the development of tooth demineralization and/or caries (24). Furthermore, $48 \%$ of the subjects with a moderate or normal SFR in the current study were in the high caries risk group, with a mean DMFT score of 5.48 , which was greater than the mean DMFT score of 4.89 calculated for the whole study group. The latter finding 
also confirmed that hyposalivation was not required for the development of caries, implying that a more reliable diagnostic tool is required for the assessment of caries risk. Other limitations on the use of SFR for this purpose include the requirement for full co-operation of the child, who has to sit still for quite some time, the possible impact of increases or decreases in mastication on saliva output, the tedious and invasive nature of the technique used for the collection of saliva from individual glands and the challenging nature of measuring SFR in young or physically or mentally handicapped children (28-30). Therefore, no currently available test is sufficiently specific and sensitive for the assessment of caries risk based on the examination of the saliva samples alone.

Conversely, the association between the vascular supply and functional activity of the salivary glands has been an attractive focus of research. Most recently, Stamers et al. (31) proposed an appropriate experimental model for the investigation of ischemic diseases of the salivary glands and investigated the functional activity of the salivary glands in reduced and restored arterial flow conditions. The functional activity of rabbit salivary glands after ligature of the common carotid artery was strongly depressed, and it was concluded that the arterial blood supply played an important role in the functional activity of the salivary glands. In a similar fashion, a reduction in submandibular blood flow was found to be associated with a decreased flow of saliva $(32,33)$. In other animal studies, the flow of saliva was found to be related linearly to blood flow to the submandibular gland $(34,35)$. The data from the literature above suggest that imaging tools relying on the quantitative assessment of the vascular supply of the salivary glands may have a role in clarifying the pathophysiology of various clinical situations associated with aberrations in saliva production.

US is usually the initial imaging study used for the diagnostic evaluation of salivary glands in children, given that most of these lesions are benign and are shown clearly on US (36). CDUS is a non-invasive technique that has proved useful in detecting blood vessel wall abnormalities and measuring blood flow velocity in many pathological states (18). Although the salivary glands have a rich and complex vasculature and intense hyperemic responses are associated with the secretion of saliva, little attention has been paid to CDUS in this field, and reports on the functional imaging of the salivary glands are scarce. During CDUS examinations, maximal stimulation of salivation can be elicited by lemon juice, resulting in dramatic vascular changes $(15,18)$. Specifically, a marked increase in color signals and the development of aliasing artifacts within the vessels, due to increasing flow velocities, occur abruptly on administration of lemon. Such physiological changes can also be detected by spectral analysis, with a decrease in RI and a marked increase in PSV. In addition to similar results obtained for these two spectral parameters, a decrease in PI and an increase in FV were detected in the current study, as expected.

In a previous study, the arterial and venous flow to the submandibular gland increased markedly in response to secretory stimulation by lemon juice in normal subjects (37). Notably, the secretory and vasodilatory responses to gustatory stimulation are mainly mediated by nervous reflex mechanisms $(38,39)$. However, there has been controversy regarding the direct relationship between secretion and blood flow without nervous intervention (38-40). In another study conducted on a group of healthy volunteers, in which the changes in blood flow to the submandibular gland were compared to salivary secretion after stimulation with a slice of lemon, the prestimulation and poststimulation arterial blood flows were evaluated with high-frequency CDUS and spectral analysis; the color signals and velocities increased, and the PI decreased after stimulation (41). Based on the close correlation between the increase in minimum velocity and that of salivary secretion, the authors concluded that CDUS is useful in analyzing changes in blood flow to the submandibular gland caused by gustatory stimulation (41). Contrary to the current study, in which the poststimulatory differences for MSV, PI, RI, and $\mathrm{FV}$ for the submandibular vascular supply showed markedly significant correlations with the SFR, the authors of the above cited study noted a significant correlation only between the minimum velocity and the difference in SFR induced by lemon stimulation.
The difference between the findings of the two studies can be attributed to the different methods applied to saliva collection, the improvements in US technology over time and the different age groups of the subjects. More importantly, the major methodological difference between the two studies was that only the submandibular gland was examined by the authors of that study, whereas the current study was based on examination of the parotid gland as well.

Notably, the submandibular glands, with a percentage of $65 \%$, are the major contributors to resting unstimulated saliva, whereas high stimulated flow rates drastically change the percentage contributions from each gland, with the parotid contributing more than $50 \%$ of total salivary secretions (42). We think that the incorporation of an evaluation for the parotid component of whole saliva secretion and the vascular supply to the parotid gland, rather than relying solely on the evaluation of the submandibular gland, would improve the results of the diagnostic utility of CDUS. The aforementioned correlations, confirming the association between spectral Doppler parameters and SFR, are supported by the significant differences in the mean values of relevant spectral parameters among different SFR groups. Finally, the significant correlations between the combined $\mathrm{dmft}$ and DMFT scores and delta values for MSV and PI obtained at the external carotid artery, as well as the significant differences between the low and high caries risk groups for the same parameters obtained at the same location, could be directly used for demonstrating the association between caries risk and spectral parameters. Nevertheless, a relatively lower degree of significance for the aforementioned correlations of MSV and PI, as well the paucity of the number of spectral parameters shown to have significant correlations with SFR, suggests that interaction with other risk factors that might have an impact on spectral waveforms should also be considered to accurately predict caries occurence. Moreover, the presence of subjects with overlapping values for the spectral indices and the relevant delta values still presents a limitation to the appropriate interpretation of the findings and their use in clinical practice. 
CDUS can be regarded as an alternative tool for caries risk assessment, not only for otherwise healthy children, but also for those children with defective salivary secretion due to various chronic inflammatory diseases that affect the pediatric population. In a study of patients with Sjögren's syndrome, the facial arteries of healthy controls at rest exhibited a biphasic waveform characterized by a high systolic peak and a prominent second peak of compliance that were followed by low diastolic flow; these participants responded to stimulation of salivary secretion by decreasing RI and PI, representing increased blood flow to the submandibular gland (43). In contrast, the waveform of patients with Sjögren's syndrome was more uniform compared with the healthy controls, substantiated by decreased resistive and pulsatility indices and suggesting a hyperemic state of the downstream vascular bed. The facial artery of patients with Sjögren's syndrome responded insufficiently to the stimulation, with the magnitude of changes in RI and PI being significantly less than that of the controls (43). The authors concluded that blood inflow responses to secretory stimulation may be defective in the salivary glands of patients with Sjögren's syndrome (43). In another study of patients with Sjögren's syndrome, of the CDUS parameters, only PSV was influenced by the degree of chronic inflammation, suggesting that PSV, rather than RI, may reflect the vascular changes that occur in salivary glands during the course of autoimmune diseases, such as Sjögren's syndrome (18). Similarly, the sonographic changes in Sjögren's syndrome were reported by Gritzmann et al. (44) to be correlated with histological destruction, and stimulation-induced hyperemia was noted to be impaired in fibrotic cases, contrary to the hypervascularization detected by CDUS in acute forms. In this regard, the mean values and poststimulatory differences of the spectral indices calculated in the current study may be regarded as reference values for future studies focusing on the evaluation of the involvement of the salivary glands in various systemic or autoimmune diseases.

Based on the findings of the current study, we think that the results of the current investigation may provide a basis on which to develop a system for conducting an early diagnosis of carioreceptivity, which may be particularly helpful in implementing caries prevention in the pediatric population. However, future research focusing on the correlation between spectral Doppler parameters and caries risk factors other than DMFT is required. In conclusion, we suggest that CDUS, non-invasively enabling the evaluation of changes in blood perfusion in the salivary glands during salivary stimulation, is a promising tool for the assessment of caries risk in children.

\section{Conflict of interest disclosure}

Authors declared no conflicts of interest.

\section{References}

1. Caufield PW, Griffen AL. Dental caries. An infectious and transmissible disease. Pediatr Clin North Am 2000; 47:10011019.

2. Shifa S, Muthu MS, Amarlal D, Rathna Prabhu V. Antitative assessment of IgA levels in the unstimulated whole saliva of caries-free and caries-active children. J Indian Soc Pedod Prev Dent 2008; 26:158-161.

3. Lenander-Lumikari M, Loimaranta V. Saliva and dental caries. Adv Dent Res 2000; 14:40-47.

4. Cheaib Z, Ganss C, Lamanda A, Turgut MD, Lussi A. Comparison of three striptype tests and two laboratory methods for salivary buffering analysis. Odontology 2012; 100:67-75.

5. Vitorino R, Calheiros-Lobo MJ, Duarte JA, Domingues P, Amado F. Salivary clinical data and dental caries susceptibility: is there a relationship? Bull Group Int Rech Sci Stomatol Odontol 2006; 47:27-33.

6. Lamanda A, Cheaib Z, Turgut MD, Lussi A. Protein buffering in model systems and in whole human saliva. PLoS One 2007; 2:e263.

7. Vanderas AP. Bacteriologic and nonbacteriologic criteria for identifying individuals at high risk of developing dental caries: a review. J Public Health Dent 1986; 46:106113.

8. Zoccola GC, Zaffalon L, Careglio A, Sapino S, Gatto V. Caries receptivity: a modern diagnostic protocol. II. The most important tests. Minerva Stomatol 1991; 40:329-337.

9. Tenovuo J. Salivary parameters of relevance for assessing caries activity in individuals and populations. Community Dent Oral Epidemiol 1997; 25:82-86.

10. Chaushu S, Becker A, Chaushu G, Shapira J. Stimulated parotid salivary flow rate in patients with Down syndrome. Spec Care Dentist 2002; 22:41-44.

11. Edgar WM. Saliva: its secretion, composition and functions. Br Dent J 1992; 172:305-312.

12. Dawes C, Kubieniec K. The effects of prolonged gum chewing on salivary flow rate and composition. Arch Oral Biol 2004; 49:665-669.
13. Nauntofte B, Tenovuo JO, Lagerlöf F. Secretion and composition of saliva. In: Fejerskov O, Kidd EAM, eds. Dental caries. The disease and its clinical management. Denmark: Blackwell Munksgaard, 2003; 7-27.

14. Humphrey SP, Williamson RT. A review of saliva: normal composition, flow, and function. J Prosthet Dent 2001; 85:162-169.

15. Martinoli C, Derchi LE, Solbiati L, Rizzatto G, Silvestri E, Giannoni M. Color Doppler sonography of salivary glands. AJR Am J Roentgenol 1994; 163:933-941.

16. Lung MA. Variations in blood flow on mandibular glandular secretion to autonomic nervous stimulations in anaesthetized dogs. J Physiol 1990; 431:479-493.

17. Thakor AS, Brown CN, Edwards AV. Effects of prolonged reduction in blood flow on submandibular secretory function in anesthetized sheep. J Appl Physiol 2003; 95:751-757.

18. Carotti M, Salaffi F, Manganelli P, Argalia G. Ultrasonography and colour doppler sonography of salivary glands in primary Sjogren's syndrome. Clin Rheumatol 2001; 20:213-219.

19. Dawes C. Rhythms in salivary flow rate and composition. Int $\mathrm{J}$ Chronobiology 1974; 2:253-279.

20. Gökalp S, Güçiz DB, Tekçiçek M, Berberoğlu A, Ünlüer Ş. Oral health profile of children aged five, twelve and fifteen years in Turkey-2004. Hacettepe Dişhekimliği Fakültesi Dergisi 2007; 31:3-10.

21. Arneberg P, Storhaug K, Sandvik L. Effect of a slow release transcutaneous scopolamine application on salivary flow, $\mathrm{pH}$, buffering action, and salivary levels of Streptococcus mutans and lactobacilli. Scand J Dent Res 1989; 97:408-414.

22. Parvinen T, Larmas $M$. The relation of stimulated salivary flow rate and $\mathrm{pH}$ to Lactobacillus and yeast concentrations in saliva. J Dent Res 1981; 60:1929-1935.

23. Younger $H$, Harrison $T$, Streckfus C. Relationship among stimulated whole, glandular salivary flow rates, and root caries prevalence in an elderly population: a preliminary study. Spec Care Dentist 1998; 18:156-163.

24. Bardow A, Nyvad B, Nauntofte B. Relationships between medication intake, complaints of dry mouth, salivary flow rate and composition, and the rate of tooth demineralization in situ. Arch Oral Biol 2001; 46:413-423.

25. Pajukoski H, Meurman JH, SnellmanGröhn S, Keinanen S, Sulkava R. Salivary flow and composition in elderly patients referred to an acute care geriatric ward. Oral Surg Oral Med Oral Pathol Oral Radiol Endod 1997; 84:265-271.

26. Ericsson Y, Hardwick L. Individual diagnosis, prognosis and counselling for caries prevention. Caries Res 1978; 12:94-102.

27. Ghezzi EM, Lange LA, Ship JA. Determination of variation of stimulated salivary flow rates. J Dent Res 2000; 79:1874-1878.

28. Andersson R, Arvidsson E, Crossner CG, Holm AK, Mansson B. The flow rate, $\mathrm{pH}$ and buffer effect of mixed saliva in children. J Int Assoc Dent Child 1974; 5:5-12. 
29. Ben-Aryeh H, Fisher M, Szargel R, Laufer D. Composition of whole unstimulated saliva of healthy children: changes with age. Arch Oral Biol 1990; 35:929-931.

30. Dodds MW, Johnson DA, Yeh CK. Health benefits of saliva: a review. J Dent 2005; 33:223-233.

31. Stamers K, Skagers A, Pastars K, Tomisheva N, Ratniece M. Functional activity of rabbit salivary glands in reduced and restored regional arterial blood supply conditions. Stomatologija 2010; 12:28-32.

32. Thakor AS, Brown CN, Edwards AV. Effects of prolonged reduction in blood flow on submandibular secretory function in anesthetized sheep. J Appl Physiol 2003; 95:751-757.

33. Rourke K, Edwards AV. Submandibular secretory and vascular responses to stimulation of the parasympathetic innervation in anesthetized cats. J Appl Physiol 2000; 89:1964-1970.

34. Hanna SJ, Brelen ME, Edwards AV. Effects of reducing submandibular blood flow on secretory responses to parasympathetic stimulation in anaesthetized cats. Exp Physiol 1999; 84:677-687.
35. Harrison AP, Cunningham ME, Edwards AV. Effects of endothelin on submandibular salivary responses to parasympathetic stimulation in anaesthetized sheep. Auton Neurosci 2002; 99:47-53.

36. Garcia CJ, Flores PA, Arce JD, Chuaqui B, Schwartz DS. Ultrasonography in the study of salivary gland lesions in children. Pediatr Radiol 1998; 28:418-425.

37. Arita T, Fujita T, Yoneshiro S, et al Usefulness of color Doppler flow imaging for blood flow in salivary gland. Nihon Igaku Hoshasen Gakkai Zasshi 1993; 53:865-867.

38. Izumi H, Karita K. Parasympatheticmediated reflex salivation and vasodilatation in the cat submandibular gland. Am J Physiol 1994; 267:747-753.

39. Lung MA. Mechanisms of sympathetic enhancement and inhibition of parasympathetically induced salivary secretion in anaesthetized dogs. Br J Pharmacol 1994; 112:411-416.

40. Izumi H, Karita K. The vasodilator and secretory effects elicited by sympathetic nerve stimulation in cat submandibular gland. J Auton Nerv Syst 1994; 48:143-151.
41. Ariji Y, Yuasa H, Ariji E. High-frequency color Doppler sonography of the submandibular gland: relationship between salivary secretion and blood flow. Oral Surg Oral Med Oral Pathol Oral Radiol Endod 1998; 86:476-481.

42. Edgar WM. Saliva and dental health. Clinical implications of saliva: report of a consensus meeting. Br Dent J 1990; 169:96-98.

43. Chikui T, Yonetsu K, Izumi M, Eguchi K, Nakamura T. Abnormal blood flow to the submandibular glands of patients with Sjogren's syndrome: doppler waveform analysis. J Rheumatol 2000; 27:1222-1228.

44. Gritzmann N, Rettenbacher T, Hollerweger A, Macheiner P, Hubner E. Sonography of the salivary glands. Eur Radiol 2003; 13:964-975. 